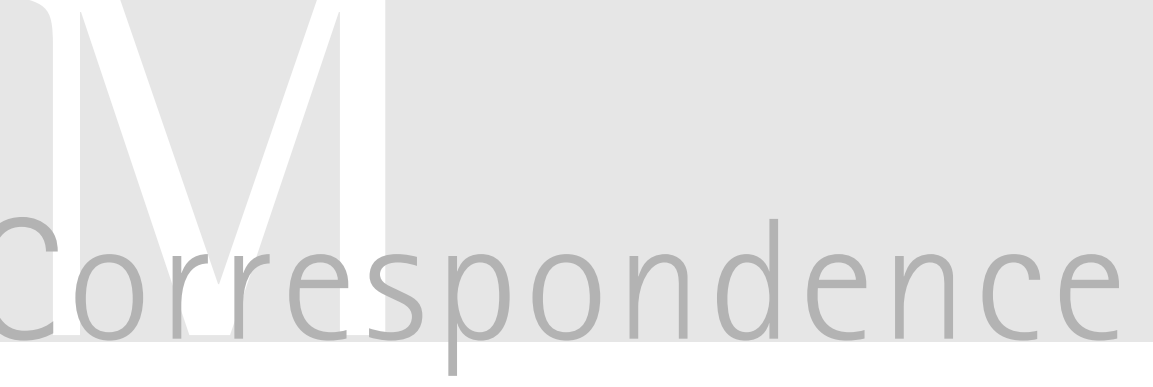

\title{
Mapping the fitness of Mycobacterium tuberculosis strains: a complex picture
}

Acquisition of antibiotic resistance in Mycobacterium tuberculosis has been shown to incur a fitness cost; the size of which depends on the position and nature of the mutation. The frequency at which resistant $M$. tuberculosis strains are isolated in vivo has been correlated with the in vitro fitness of the different corresponding mutants in laboratory strains. In $M$. tuberculosis certain resistant genotypes, such as the Ser315Thr mutation in katG conferring isoniazid resistance or Ser531Leu in $r p o B$ conferring rifampicin resistance, occur with minimal or no reduction in fitness (Billington et al., 1999; Pym et al., 2002). These mutations are the ones most commonly found in clinical isolates of resistant M. tuberculosis, which suggests that they have a higher relative fitness on initial isolation (Billington et al., 1999; Ramaswamy \& Musser, 1998; Van Soolingen et al., 2000). The genetic background of the organism is an important contributor to its fitness (Toungoussova et al., 2004). This has been investigated by relating $r p o B$ mutation His526Asp in two different M. tuberculosis strain backgrounds with their respective biological fitness, which suggests that the cost of resistance mutations may differ according to strain phylogeny (Gagneux et al., 2006). The acquisition of multiple drug resistance (MDR) coupled with a fitness deficit does not necessarily mean that the strain has lost its capability to cause disease and remain virulent. Rather the strain has adapted to the environment of the individual host (Davies et al., 2000).

To investigate the hypothesis that the fitness cost of drug resistance in clinical strains is related to their resistance mutation we developed a rapid and reproducible method of fitness estimation. Using generation time as an indicator of fitness, we used an automated liquid culture system, the MB/BacT ALERT 3D, to investigate the fitness of 18 clinical isolates of $M$. tuberculosis from 13 patients at the Royal Free Hospital (London, UK) with different resistance phenotypes (Table 1). The Erdman and H37Rv laboratory strains were used as controls. An aliquot $(0.5 \mathrm{ml})$ from a primary culture was inoculated into a BacT/Alert MP liquid culture bottle (bioMérieux) and once this signalled positive in the $\mathrm{MB} / \mathrm{BacT}$ ALERT 3D system, $0.5 \mathrm{ml}$ was removed, and $1: 10$ and 1:10000 dilutions were prepared. A $0.5 \mathrm{ml}$ aliquot of these dilutions was inoculated, in triplicate, into fresh BacT/ Alert MP culture bottles. A modified Youmans and Youmans method was used to calculate the generation time (Pope et al., 2008; Youmans \& Youmans, 1949). This process was repeated on at least three occasions for each strain. Statistical differences between isolates were analysed by one-way ANOVA using GraphPad Prism version 4.02 for Windows with a $P$ value $<0.05$. The supernatant from the heat-killed harvested culture was used as a template for PCR. A 411 bp fragment of $r p o B$ that included the RRDR (rifampicin resistance determining region) was amplified with the primers described by Telenti et al. (1993). Resistance to isoniazid was investigated in $k a t G$ and inhA. The primers used to amplify $k a t G$ targeted a $185 \mathrm{bp}$ fragment that included codon 315 (5'-GTC ACA CTT TCG GTA AGA CC-3', 5'-TTG TCC CAT TTC GTC GGG-3'), and a 296 bp product that included codon $463\left(5^{\prime}\right.$-GCG AAG CCG AGA TTG CCA GC-3' ', 5' -ACA GCC ACC GAG CAC GAC GA-3'). A 248 bp fragment of the inhA promoter was amplified using the primers described by Telenti et al. (1997). Sequencing was performed using the dideoxy method as described by Jenkins et al. (2005).

It is notable that the laboratory adapted Erdman strain had the shortest generation time (14.3 h; Table 1). For the purposes of determining significant differences, all sensitive clinical isolates (06:083, 06:092, $06: 095)$ were included in the analysis. The mono-resistant isolates, $06: 120,06: 181$ and $07: 118$ did not have statistically slower generation times compared to sensitive isolates $(06: 083,06: 092$, 06 : 095). However, a streptomycin monoresistant isolate, $06: 216$, had a significantly slower generation time (27.2 h) compared to sensitive clinical isolates ( $P$ value $<0.05)$. The isoniazidand streptomycin-resistant isolates, $07: 145$ and $07: 146$ (mean generation time for the two isolates $19.6 \mathrm{~h}$ ) and the isoniazid- and ethionamide-resistant isolates, $07: 112,07: 116$ and $07: 126$ (mean generation time for the three isolates $16.5 \mathrm{~h}$ ) did not achieve statistical significance compared to the sensitive clinical isolates (mean generation time for the three isolates $19.5 \mathrm{~h}$ ).

It was observed that some clinical MDR isolates $(05: 371,06: 209)$ had statistically significant slower generation times compared to sensitive clinical isolates. Additional pyrazinamide and ethambutol resistances, as well as the previously unreported $r p o B$ mutation Thr480Ser, may be responsible for the slow growth of isolate $05: 371$. It may not be just this specific genotype that is contributing to the slower generation time but the phenotype may represent further adaptations. Additional mutations were noted in isolate $06: 209$, a mutation in $r p o B$ Ser531Leu, and a double mutation in kat $G$, Ser315Thr and Arg463Leu. The polymorphism at codon 463 is not associated with isoniazid resistance but has been used to assign isolates to genetic group 1 (Sreevatsan et al., 1997).

Multiple isolates $07: 171,07: 179$ and 07:198 from the same patient with MDR were resistant to pyrazinamide, and had a generation time that was not significantly different to sensitive clinical isolates. They had the Asp516Tyr mutation in $r p o B$ and the Ser315Thr mutation in katG. The MDR isolate 06:042, which was resistant to rifampicin and isoniazid, contained an Asp516Val mutation in $r p o B$, the fourth most common in practice $(\sim 8 \%)$ 
Table 1. The mean generation time including SEM, and the resistance genotypes of a panel of laboratory and clinical strains of $M$. tuberculosis

The laboratory isolates H37Rv and Erdman, and clinical isolates 06:083, 06:092 and 06:095, are drug sensitive. Shading of the same intensity indicates multiple isolates from the same patient.

\begin{tabular}{|c|c|c|c|c|c|c|c|}
\hline \multirow[t]{2}{*}{ Isolate } & \multirow[t]{2}{*}{ Resistance phenotype } & \multirow{2}{*}{$\begin{array}{c}\text { No. of } \\
\text { experiments }\end{array}$} & \multirow{2}{*}{$\begin{array}{c}\text { Mean generation } \\
\text { time }(\mathrm{h})\end{array}$} & \multirow[t]{2}{*}{ SEM } & \multicolumn{3}{|c|}{ Resistance mutation } \\
\hline & & & & & rров & katG & $\operatorname{inh} A$ \\
\hline Erdman & - & 3 & 14.3 & 0.87 & - & - & - \\
\hline H37Rv & - & 4 & 15.9 & 0.73 & - & - & - \\
\hline $06: 083$ & - & 3 & 23.7 & 3.85 & - & - & - \\
\hline $06: 092$ & - & 3 & 18.3 & 2.12 & - & - & - \\
\hline $06: 095$ & - & 3 & 16.5 & 1.98 & - & - & - \\
\hline $07: 118$ & INH & 5 & 19.1 & 1.01 & - & Ser315Thr & - \\
\hline $06: 120$ & INH & 3 & 19.6 & 2.29 & - & None & None \\
\hline $06: 181$ & PZN & 6 & 18 & 0.97 & - & - & - \\
\hline $06: 216$ & STR & 6 & 27.2 & 1.59 & - & - & - \\
\hline $07: 126$ & INH, ETH & 3 & 14.4 & 1.89 & - & None & $-15 \mathrm{C} \rightarrow \mathrm{T}$ \\
\hline $07: 116$ & INH, ETH & 6 & 16.4 & 1.22 & - & None & $-15 \mathrm{C} \rightarrow \mathrm{T}$ \\
\hline $07: 112$ & INH, ETH & 4 & 18.6 & 0.79 & - & None & $-15 \mathrm{C} \rightarrow \mathrm{T}$ \\
\hline $07: 146$ & INH, STR & 3 & 17.9 & 1.45 & - & Ser315Thr, Arg463Leu & - \\
\hline $07: 145$ & INH, STR & 7 & 21.3 & 2.51 & - & Ser315Thr, Arg463Leu & - \\
\hline $06: 042$ & INH, RIF & 3 & 24.8 & 5.61 & Asp516Val & None & None \\
\hline $06: 209$ & INH, RIF & 4 & 57.7 & 7.91 & Ser531Leu & Ser315Thr, Arg463Leu & - \\
\hline $07: 198$ & INH, RIF, PZN & 3 & 22.2 & 1.37 & Asp516Tyr & Ser315Thr & - \\
\hline $07: 171$ & INH, RIF, PZN & 3 & 31.3 & 2.02 & Asp516Tyr & Ser315Thr & - \\
\hline $07: 179$ & INH, RIF, PZN & 3 & 31.9 & 8.33 & Asp516Tyr & Ser315Thr & - \\
\hline $05: 371$ & INH, RIF, EMB, PZN & 3 & 67.9 & 11.42 & Thr480Ser & None & None \\
\hline
\end{tabular}

EMB, Ethambutol; ETH, ethionamide; INH, isoniazid; PZN, pyrazinamide; RIF, rifampicin; STR, streptomycin.

(O’Sullivan et al., 2005; Ramaswamy \& Musser, 1998), and did not have a significant fitness deficit compared to sensitive clinical isolates. No isoniazidresistance-conferring mutation was identified; this was the same as for isolate $06: 120$ and $05: 371$, even though the sequences that were approximately $75 \%$ likely to contain the isoniazidresistance-determining mutation were investigated.

These drug-resistant isolates were collected over a 3 year period and as expected with limited IS6110 RFLP typing data, no clusters were identified (data not shown). However, isolates $07: 116$ and $07: 118$ had a similarity of $76 \%$ as measured by the Dice coefficient. These isolates had a different phenotype and drug-resistance genotype; $07: 116$ had a mutation in promoter region of inhA $(-15 \mathrm{C} \longrightarrow \mathrm{T})$ and $07: 118$ had a mutation Ser315Thr, conferring isoniazid resistance, yet the mean generation time for each isolate was not statistically different.
Isoniazid-resistant isolates with the most commonly isolated Ser315Thr mutation did not have a significant fitness deficit compared to sensitive clinical isolates. This finding mirrors a report by Van Soolingen and colleagues who showed that $k a t G$ codon 315 mutants lead to secondary cases of tuberculosis as often as isoniazidsusceptible strains, implying no significant loss of fitness (Van Soolingen et al., 2000). Also certain rpsL mutations in streptomycin-resistant isolates of $M$. tuberculosis appear to confer no fitness cost (Böttger et al., 1998).

We have demonstrated that strains with mutations previously shown to have low or no cost as measured in vitro and by frequency of clinical isolation had a longer generation time indicating the effect of either the genetic background or the transmission history, which may be outweighed by the fitness effect of the $r p o B$ mutation. Over time, strains from the same patient had a decreased generation time $(07: 198)$, from initial isolation
(07:171) but it was not statistically significant. Although, if the time between sample isolations from the patient had been greater, we may have observed significant differences.

Our results show that fitness of an isolate is complex, depending on its drug resistance profile and the genetic context of the resistance. Even though this is a study that has limited isolate numbers that are heterogeneous with respect to their genetic background, it could still be observed that the majority of rifampicin-resistant strains are significantly less fit, as measured by generation time, than strains with resistance other than that to rifampicin. The differences demonstrated in clinical isolates may be due to compensatory mutations ameliorating the fitness deficit. This may occur by adaptation to the host as we showed in our study of the fitness of an MDR strain transmitted during an outbreak (Gillespie et al., 2002). The limited typing data for these isolates would suggest that the overall genotype of the strain is significant 
in terms of its fitness not just its drug resistance mutation. Further work should involve analysing a larger more-defined group of clinical isolates.

M. tuberculosis lives in a complex environment within the human host where numerous adaptive pressures are applied, of which antibiotic selection is but one. The data that we have reported previously (Gillespie et al., 2002) and new evidence presented here suggest that the impact of transmission and the acquisition of additional resistance determinants complicate predictions about isolate fitness. It has been proposed that mathematical models can predict the epidemic potential of MDR strains (Gagneux et al., 2006), but M. tuberculosis takes multiple routes through the adaptive landscape and thus such models must consider the full range of factors influencing fitness.

\section{Acknowledgements}

This work was supported by the European Union Framework 6 grant LSHM-CT-20058152 EAR. We thank R. J. Shorten (University College London, London, UK) for performing the RFLP typing.

\section{M. O'Sullivan, ${ }^{1, \dagger}$ T. D. McHugh ${ }^{1}$ and S. H. Gillespie ${ }^{1,2}$}

${ }^{1}$ Department of Infection, Royal Free Campus, University College London, Rowland Hill Street, Hampstead, London NW3 2PF, UK

${ }^{2}$ Health Protection Agency, Regional Microbiology Network, Holborn Gate, London WC1V 7PP, UK
Correspondence: D. M. O'Sullivan

(denise.o'sullivan@Ishtm.ac.uk)

tPresent address: Department of Pathogen Molecular Biology, London School of Hygiene and Tropical Medicine, Keppel Street, London WC1E 7HT, UK.

Billington, O. J., McHugh, T. D. \& Gillespie, S. H. (1999). Physiological cost of rifampin resistance induced in vitro in Mycobacterium tuberculosis. Antimicrob Agents Chemother 43, 1866-1869.

Böttger, E. C., Springer, B., Pletschette, M. \& Sander, P. (1998). Fitness of antibiotic-resistant microorganisms and compensatory mutations. Nat Med 4, 1343-1344.

Davies, A. P., Billington, O. J., Bannister, B. A., Weir, W. R., McHugh, T. D. \& Gillespie, S. H. (2000). Comparison of fitness of two isolates of Mycobacterium tuberculosis, one of which had developed multi-drug resistance during the course of treatment. J Infect 41, 184-187.

Gagneux, S., Long, C. D., Small, P. M., Van, T., Schoolnik, G. K. \& Bohannan, B. J. M. (2006). The competitive cost of antibiotic resistance in $\mathrm{Myco}-$ bacterium tuberculosis. Science 312, 1944-1946.

Gillespie, S. H., Billington, O. J., Breathnach, A. \& McHugh, T. D. (2002). Multiple drug-resistant Mycobacterium tuberculosis: evidence for changing fitness following passage through human hosts. Microb Drug Resist 8, 273-279.

Jenkins, C., Claxton, A. P., Shorten, R. J., McHugh, T. D. \& Gillespie, S. H. (2005). Rifampicin resistance in tuberculosis outbreak, London, England. Emerg Infect Dis 11, 931-934.

O'Sullivan, D. M., McHugh, T. D. \& Gillespie,

S. H. (2005). Analysis of $r p o B$ and $p n c A$ mutations in the published literature: an insight into the role of oxidative stress in Mycobacterium tuberculosis evolution? J Antimicrob Chemother 55, 674-679.

Pope, C. F., Gillespie, S. H., Pratten, J. R. \& McHugh, T. D. (2008). Fluoroquinoloneresistant mutants of Burkholderia cepacia. Antimicrob Agents Chemother 52, 1201-1203.
Pym, A. S., Saint-Joanis, B. \& Cole, S. T. (2002). Effect of kat $G$ mutations on the virulence of Mycobacterium tuberculosis and the implication for transmission in humans. Infect Immun 70, 4955-4960.

Ramaswamy, S. \& Musser, J. M. (1998). Molecular genetic basis of antimicrobial agent resistance in Mycobacterium tuberculosis: 1998 update. Tuber Lung Dis 79, 3-29.

Sreevatsan, S., Pan, X., Stockbauer, K. E., Connell, N. D., Kreiswirth, B. N., Whittam, T. S. \& Musser, J. M. (1997). Restricted structural gene polymorphism in the Mycobacterium tuberculosis complex indicates evolutionarily recent global dissemination. Proc Natl Acad Sci U S A 94, 9869-9874.

Telenti, A., Imboden, P., Marchesi, F., Matter, L., Schopfer, K., Bodmer, T., Lowrie, D., Colston, M. J. \& Cole, S. (1993). Detection of rifampicinresistance mutations in Mycobacterium tuberculosis. Lancet 341, 647-651.

Telenti, A., Honore, N., Bernasconi, C., March, J., Ortega, A., Heym, B., Takiff, H. E. \& Cole, S. T. (1997). Genotypic assessment of isoniazid and rifampin resistance in Mycobacterium tuberculosis: a blind study at reference laboratory level. J Clin Microbiol 35, 719-723.

Toungoussova, O. S., Caugant, D. A., Sandven, P., Mariandyshev, A. O. \& Bjune, G. (2004). Impact of drug resistance on fitness of Mycobacterium tuberculosis strains of the W-Beijing genotype. FEMS Immunol Med Microbiol 42, 281-290.

Van Soolingen, D., De Haas, P. E. W., Van Doorn, R., Kuijper, E., Rinder, H. \& Borgdorff, M. W. (2000). Mutations at amino acid position 315 of the kat $G$ gene are associated with highlevel resistance to isoniazid, other drug resistance, and successful transmission of Mycobacterium tuberculosis in The Netherlands. $J$ Infect Dis 182, 1788-1790.

Youmans, G. P. \& Youmans, A. S. (1949). A method for the determination of the rate of growth of tubercle bacilli by the use of small inocula. J Bacteriol 58, 247-255. 